\title{
Long-term follow-up of buserelin-induced enteric neuropathy in rats
}

\author{
ANETTE JÖNSSON $^{1 *}$, ELIN SAND $^{1,2^{*}}$, EVA EKBLAD $^{2 *}$ and BODIL OHLSSON ${ }^{1}$ \\ ${ }^{1}$ Department of Clinical Sciences, Division of Internal Medicine, Skåne University Hospital, \\ Lund University, 20502 Malmö; ${ }^{2}$ Department of Experimental Medical Science, \\ Neurogastroenterology Unit, BMC B11, Lund University, 22184 Lund, Sweden
}

Received November 27, 2015; Accepted February 15, 2016

DOI: $10.3892 / \mathrm{mmr} .2016 .4968$

\begin{abstract}
A few patients have been shown to develop severe abdominal pain and gastrointestinal dysmotility during treatment with gonadotropin-releasing hormone $(\mathrm{GnRH})$ analogs. A rat model of enteric neuropathy has been developed by administration of the GnRH analog buserelin to rats. Loss of enteric neurons and ganglioneuritis throughout the gastrointestinal tract has been described, without other histopathological changes. The aim of the present study was to investigate the long-term effects of this rat model on body weight, and on morphology and inflammatory changes in the gastrointestinal tract. Rats were administered subcutaneous injections of buserelin or saline once daily for 5 days and allowed to recover for 3 weeks. This regimen was repeated four times. The rats were weighed weekly and were sacrificed 16 weeks after the fourth treatment. The bowel wall was measured by morphometry, and the presence of enteric neurons, mast cells, eosinophils and T-lymphocytes was evaluated. Buserelin-treated rats were shown to have a lower body weight at sacrifice, as compared with the controls $(\mathrm{P}<0.05)$. Compared with controls, buserelin treatment caused loss of myenteric neurons in the ileum and colon $(\mathrm{P}<0.01)$, a thinner circular muscle layer in ileum $(\mathrm{P}<0.05)$ and longitudinal muscle layer in colon $(\mathrm{P}<0.05)$, increased number of eosinophils in the submucosa of the ileum $(\mathrm{P}<0.05)$, and an increased number of T-lymphocytes in the submucosa and circular muscle layer of the fundus $(\mathrm{P}<0.01$ and $\mathrm{P}<0.05$, respectively) and circular muscle layer of the colon $(\mathrm{P}<0.05)$.
\end{abstract}

Correspondence to: Professor Bodil Ohlsson, Department of Clinical Sciences, Division of Internal Medicine, Skåne University Hospital, Lund University, 32 Inga Marie Nilsson Street, 20502 Malmö, Sweden

E-mail: bodil.ohlsson@med.lu.se

*Contributed equally

Key words: enteric neuropathy, gonadotropin-releasing hormone, gastrointestinal tract, rat model
Mast cells were equally distributed in the two groups. Thus, long-term follow-up of buserelin-induced enteric neuropathy reveals reduced body weight, loss of myenteric neurons, thinning of muscle layers, and increased numbers of eosinophils and T-lymphocytes in the gastrointestinal tract.

\section{Introduction}

Gonadotropin-releasing hormone $(\mathrm{GnRH})$ stimulates the secretion of follicle-stimulating hormone (FSH) and luteinizing hormone (LH) from the pituitary gland, which regulate the secretion of steroid hormones, such as estrogen and progesterone (1). GnRH and LH receptors have been identified in the human and rat gastrointestinal (GI) tract (2-4), where GnRH inhibits cell proliferation $(5,6)$. LH stimulation fragments the migrating myoelectric complex in the small intestine (7) and reduces survival of enteric rat neurons in culture (8). A number of patients suffer from GI side effects during treatment with $\mathrm{GnRH}$ analogs, and a few patients develop chronic intestinal pseudo-obstruction (CIPO) or enteric dysmotility (ED) with degenerative and inflammatory changes in the GI tract $(3,9)$.

Previous rat studies have shown that $\mathrm{GnRH}$ analogs cause loss of submucosal and myenteric neurons in the fundus, ileum and colon, without any effect on body weight, circulating inflammatory biomarkers or morphometry of the bowel wall $(4,10)$. The numbers of mast cells and eosinophils were shown to be unaltered; however, signs of ganglioneuritis with infiltration of T-lymphocytes in the enteric ganglia were identified $(4,11)$. Elevated estradiol levels in the plasma and a thickened uterine muscle layer were observed, along with reduced relative numbers of neurons immunoreactive to $\mathrm{LH}$ receptors and increased imunnoreactivity of activated caspase-3 $(4,10)$. Thus, buserelin treatment in rats constitutes a rat model of enteric neuropathy, and the neuronal loss is anticipated to be mediated through elevated LH release and hyperactivation of $\mathrm{LH}$ receptor-bearing enteric neurons $(4,10,11)$, resulting in increased apoptosis $(4,12)$.

Prior results are based on studies where the animals were sacrificed shortly after the final buserelin treatment $(4,10,11)$. The aim of the present study was to investigate the long-term effect of buserelin-induced enteric neuropathy on body weight and on the GI tract regarding neuronal cell loss, morphology, and inflammatory changes. 


\section{Materials and methods}

Animals. Female Sprague-Dawley rats ( $\mathrm{n}=20$; age, 7 weeks; weight, 170-180 g), purchased from Charles River Laboratories (Sulzfeld, Germany) were used. The rats were allowed to acclimatize to the climate- and light-controlled animal facility for at least 5 days prior to experimentation. Standard rat chow (4\% fat/g) (Lactamin R36, Stockholm, Sweden) and water were supplied ad libitum. The experimental design was approved by the Animal Ethics Committee, Lund and Malmö, Sweden (M350-12, date of approval: 14.11.12). Animals were used in accordance with the European Communities Council Directive (2010/63/EU) and the Swedish Animal Welfare Act (SFS 1988:534).

Study design. Rats ( $\mathrm{n}=12)$ were administered $20 \mu \mathrm{g}$ of the GnRH analog buserelin $(1 \mathrm{mg} / \mathrm{ml})$ (Suprefact, Sanofi-Aventis, Bromma, Sweden) (dissolved in $0.2 \mathrm{ml}$ saline) subcutaneously, once daily for 5 days, followed by 3 weeks of recovery, representing one session of treatment (4). The dosage and administration of buserelin are based on previous studies which have shown reliable physiological effects in terms of uterine hypertrophy, without any adverse effects $(4,10,13)$. Control animals $(\mathrm{n}=8)$ received $0.2 \mathrm{ml}$ saline injections. The animals were weighed prior to inclusion in the study, and weekly in the morning during the study. Thirty-two weeks after the start of the study, and 4 months after the last treatment session, rats were anesthetized using chloral hydrate $(300 \mathrm{mg} / \mathrm{kg}$ body weight; C8383; Sigma-Aldrich, St. Louis, MO, USA) and killed by aorta puncture. Tissue samples from the fundus, distal ileum, proximal colon, and distal part of the uterine horn were collected and processed for histological evaluation.

Tissue preparation. The fundus/corpus region of the stomach, ileum (starting $8 \mathrm{~cm}$ proximally to the cecum and continuing in the proximal direction), and colon (starting $2 \mathrm{~cm}$ distally to the cecum and continuing in the distal direction) were opened and flattened on filter paper. One section of each gut region was fixed in Stefanini's fixative (a mixture of $2 \%$ formaldehyde (Sigma-Aldrich) and 0.2\% picric acid (Sigma-Aldrich) in phosphate buffer, $\mathrm{pH} 7.2$ ) for $22 \mathrm{~h}$ at $4^{\circ} \mathrm{C}$, and the other section and the uterine horns were fixed in $4 \%$ paraformaldehyde (Sigma-Aldrich) in $0.1 \mathrm{M}$ phosphate buffer for $22 \mathrm{~h}$ at $4^{\circ} \mathrm{C}$. Stefanini-fixed specimens were rinsed three times in Tyrode's solution containing $10 \%$ sucrose, before being orientated and mounted for cross- and longitudinal sectioning in Tissue-Tek (Sakura, Histolab, Gothenburg, Sweden), frozen on dry ice, and sectioned $(10 \mu \mathrm{m})$ in a cryostat (HM500; Microm GmbH, Walldorf, Germany). Paraformaldehyde-fixed specimens were dehydrated in ethanol, cleared in xylene, orientated for crossand longitudinal sectioning, embedded in paraffin, and sectioned $(5 \mu \mathrm{m})$. Sections were processed for immunocytochemistry and histochemistry.

Histochemistry. Morphometry was performed on scanned, deparaffinized, hydrated, and hematoxylin and eosin-stained paraffin (all Histolab Products AB, Gothenburg, Sweden) sections from cross-sectioned uterine horns and longitudinally cut GI regions using a computerized, image-analyzing system (Imagescope, Aperio ScanScope GL SS5082, Vista, CA,
USA). The myometrium and the intestinal layers of fundus, distal ileum, and proximal colon (mucosa and circular and longitudinal muscle layers) were indicated manually, and then measured using a computerized binary cursor. Mean values of 6-10 representative measurements were calculated from each rat in each region.

Immunocytochemistry. For studies on enteric neuronal survival, monoclonal mouse antibodies against human neuronal protein (HuC/D) (dilution 1:100, cat. no. A-2127, Thermo Fisher Scientific, Inc., Stockholm, Sweden) were used as general neuronal marker. Paraffin sections from the fundus, ileum and colon were deparaffinized, hydrated and subjected to antigen retrieval by boiling in citrate acid buffer $(0.01 \mathrm{M}, \mathrm{pH}$ 6; Sigma-Aldrich) in a microwave oven $(650 \mathrm{~W})$ for $7 \mathrm{~min}$ twice. The sections were cooled and washed in distilled water followed by phosphate-buffered saline (PBS)/Triton X-100 (Sigma-Aldrich). Sections were exposed to biotinylated, primary antibodies against $\mathrm{HuC} / \mathrm{D}$ at $4^{\circ} \mathrm{C}$ overnight. For visualization of biotinylated $\mathrm{HuC} / \mathrm{D}$, a VECTASTAIN ABC kit containing horseradish peroxidase (HRP) and 3,3'-diaminobenzidine tetrahydrochloride (DAB) was used (Vector Laboratories, Inc., Burlingame, CA, USA). HuC/D-immunoreactive neurons stained dark brown and were counted in submucosal and myenteric ganglia in longitudinally cut whole-wall sections using the computerized, image-analyzing system Imagescope (Aperio ScanScope). Since antigens for testing the specificity of antibodies against $\mathrm{HuC} / \mathrm{D}$ are not commercially available, omission of the primary antibodies served as a control. Numbers of $\mathrm{HuC} / \mathrm{D}$ neurons were counted in a total length of at least $20 \mathrm{~mm}$, cut at 6-9 different depths in each region and rat. Results are expressed as numbers of HuC/D-immunoreactive submucosal or myenteric neurons per $\mathrm{mm}$ region length of GI tract.

Mast cells. Toluidine blue-staining (Histolab Products AB) was performed on paraffin sections to identify mast cells in the GI tract (14). Sections from the fundus, ileum and colon were deparaffinized, hydrated, and stained with $0.1 \%$ toluidine blue in $60 \%$ ethanol for $60 \mathrm{~min}$ in room temperature. Crossand longitudinally cut, whole-wall sections were scanned and analyzed in Imagescope (Aperio ScanScope). Mucosa, submucosa, and circular and longitudinal muscle layers were evaluated separately and all mast cells were counted for at least $12 \mathrm{~mm}$, cut at 6-9 different depths from each region and rat. Results are expressed as numbers of mast cells per mm region length of GI tract.

Eosinophils. A staining procedure based on cyanide-resistant eosinophilic peroxidase (EPO) was used to identify eosinophils (15). Cryo sections from the fundus, ileum, and colon were washed in PBS buffer ( $\mathrm{pH}$ 7.2), DAB $(75 \mathrm{mg} / 100 \mathrm{ml}), \mathrm{H}_{2} \mathrm{O}_{2}(100 \mu \mathrm{l} / 100 \mathrm{ml})$ and $\mathrm{NaCN}$ $(50 \mathrm{mg} / 100 \mathrm{ml})$ for $8 \mathrm{~min}$ and then rinsed in water for $10 \mathrm{~min}$ prior to being mounted in glycerine gelatine (Kaiser, Merck K, Damstadt, Germany). Both cross- and longitudinally cut whole-wall sections were analyzed using a light microscope (Olympus BX43, with attached camera Nikon XC30, LRI instrument, Hamburg, Germany). The number of cells were evaluated separately in the mucosa, submucosa, and muscle layers of each region in a range from 0 to +++ , where 0 
indicates no cells, + indicates a few cells, ++ indicates a moderate number, and +++ indicates a high number, as previously described $(4,15)$. In regions which showed a difference between groups, eosinophils were counted for at least $30 \mathrm{~mm}$, cut at 6-9 different depths from each region and rat. Results are expressed as the number of eosinophils per $\mathrm{mm}$ or $\mathrm{cm}$ region length of GI tract.

T-lymphocytes. The presence of CD3-immunoreactive T-lymphocytes was analyzed on cross- and longitudinally cut whole-wall paraffin sections from the fundus, ileum and colon. The sections were incubated over night with primary polyclonal antibodies against CD3 raised in goat (dilution 1:1,000; cat. no. 1127, Santa Cruz Biotechnology, Inc., CA, USA). The sections were then exposed to DyLight-TM-594-conjugated donkey anti-rabbit IgG (dilution 1:1,000; Jackson ImmunoResearch Europe, Ltd., Newmarket, $\mathrm{UK}$ ) for $1 \mathrm{~h}$ and mounted in phosphate buffer:glycerol 1:1. The fluorophore was excited and T-lymphocytes were visualized at $488 \mathrm{~nm}$ using a Olympus BX43 microscope.

T-lymphocytes were separately evaluated in epithelia, mucosa, submucosa, circular and longitudinal muscle layers, and serosa of the fundus, ileum and colon, in a range from 0 to +++ , where 0 indicates no cells, + indicates few, ++ indicates a moderate number, and +++ indicates a high number, as previously described $(4,15)$. Only those regions which showed a difference between groups were counted, for at least $20 \mathrm{~mm}$ GI length, cut at 6-9 different depths from each region and rat. Results are expressed as numbers of T-lymphocytes per $\mathrm{mm}$ region length of GI tract.

Statistical analysis. Statistical analyses were performed using GraphPad Prism 5 (GraphPad Software, Inc., San Diego, CA, USA). Results are presented as the medians and spreads, expressed as the 25 th and 75 th percentile or as the mean \pm standard error of the mean. Statistical analyses were performed using unpaired Student's t-test regarding the normally distributed body weight and by Mann-Whitney U-test for other data. $\mathrm{P}<0.05$ was considered to indicate a statistically significant difference.

\section{Results}

General observations. All rats looked healthy and exhibited normal healthy behavior. One of the buserelin-treated rats presented with a subcutaneous tumor during week 22 and was sacrificed (not included in the study), leaving eight rats in the control group (C) and 11 rats in the buserelin-treated group (B). There was a significant difference in body weight between controls and buserelin-treated rats from week 23 to the end of the study (Fig. 1). At autopsy, the visceral organs were inspected, and no lesions or abnormalities were identified.

Morphology and morphometry. Hematoxylin and eosin staining of sections from the uterus, fundus, ileum and colon revealed normal histology in the controls and buserelin-treated rats. No differences in mucosal thickness were detected between the two groups. However, a thinner circular muscle layer of the fundus was observed in buserelin-treated

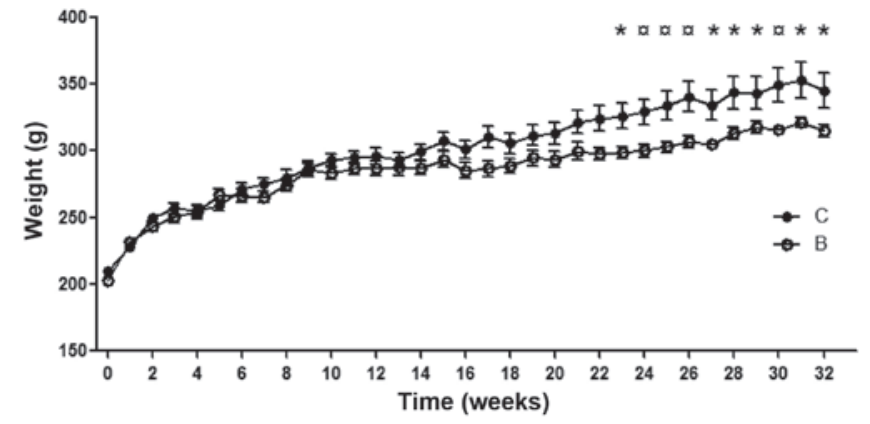

Figure 1. Body weight over time for rats treated with four sessions of saline [controls, closed circles] or buserelin (open circles). Saline or buserelin were injected once daily for 5 days at week $0,4,8$, and 12 . The treatment ended at week 16 . From the end of week 23 , the buserelin-treated rats showed a decreased body weight compared with control rats. $\mathrm{C}=8$ rats and $\mathrm{B}=11$ rats. Results are presented as the mean \pm standard error of the mean and analyzed by Student's t-test; ${ }^{*} \mathrm{P}<0.05$ and ${ }^{\circ} \mathrm{P}<0.01$, compared with control. C, control; B, buserelin.

rats, as compared with the control rats $(\mathrm{P}=0.059)$ (Fig. 2A). A thinning of the circular muscle layer in the ileum $(\mathrm{P}<0.05)$ (Fig. 2B) and the longitudinal muscle layer in colon $(\mathrm{P}<0.05)$ (Fig. 2C) were also noted in buserelin-treated rats, as compared with the controls. No difference in the thickness of uterine muscle layer was detected between the two groups (data not shown).

Neuronal survival. Neurons displaying HuC/D immunoreactivity were regularly found all along the fundus, ileum, and colon (Fig. 3A and B). In addition, the number of neurons was determined in the fundus, ileum and colon (Fig. 3C-H). No significant difference was identified in the numbers of neurons in the fundus between the controls and the buserelin-treated group (Fig. 3C and D).

In the ileum and colon, the numbers of submucosal neurons did not differ between the two groups (Fig. 3E and G), whereas reduced numbers of myenteric neurons were found in the buserelin-treated group $(\mathrm{P}<0.01)$ (Fig. 3F and $\mathrm{H})$.

Mast cells. In the fundus, numerous mast cells were identified in the submucosa and in the circular muscle layer, with a high individual variation, independent of treatment (Fig. 4A and B). In the ileum and colon, the mast cells were only found in the submucosa, without any difference between the groups (Fig. 4C and D).

Eosinophils. Eosinophils were abundant in the lamina propria of the entire GI mucosa, without any difference between the groups. Eosinophils were sparsely found in the submucosa, smooth muscle layers and serosa in the two groups. An increased number of eosinophils was detected in the submucosa of the ileum in buserelin-treated rats compared with controls $(\mathrm{P}<0.05)$ (Fig. 5A), whereas no changes were identified in the circular muscle layer or in-between the circular and longitudinal muscle layers (Fig. 5B and C).

T-lymphocytes. In the two groups, the majority of T-lymphocytes were identified in the mucosa, positioned in the lamina propria and intra-epithelially. T-lymphocytes were only rarely detected in the longitudinal muscle layer. 
A

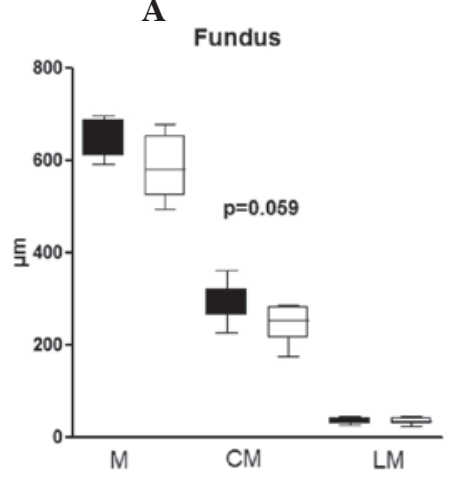

B

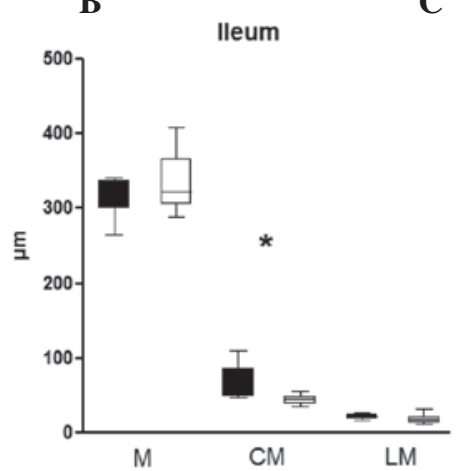

C

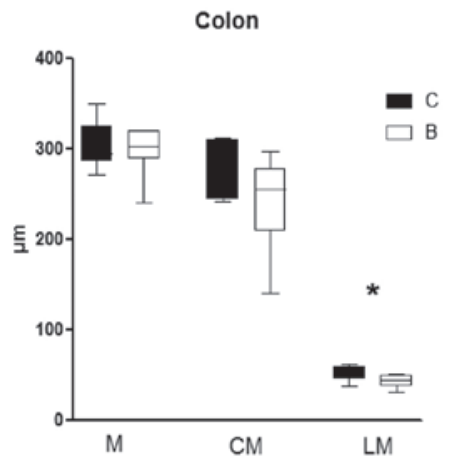

Figure 2. Measurement of the M, CM and LM in the (A) fundus, (B) ileum and $(\mathrm{C})$ colon of control rats and rats treated with buserelin. $\mathrm{C}=8$ rats and $\mathrm{B}=11$ rats. Results are presented as medians and spreads expressed as the 25 th and 75 th percentile and analyzed using a Mann-Whitney $\mathrm{U}$-test. * $\mathrm{P}<0.05$, compared with control. M, mucosa; CM, circular muscle layer; LM, longitudinal muscle layer; C, control treated with saline; B, buserelin.

A

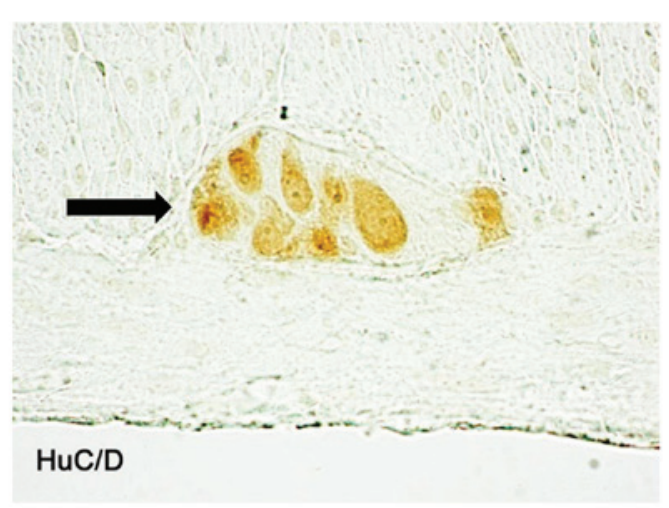

C

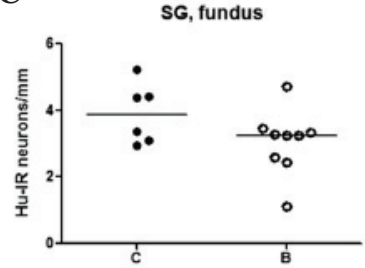

F

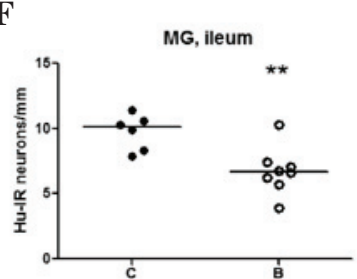

D

G
B
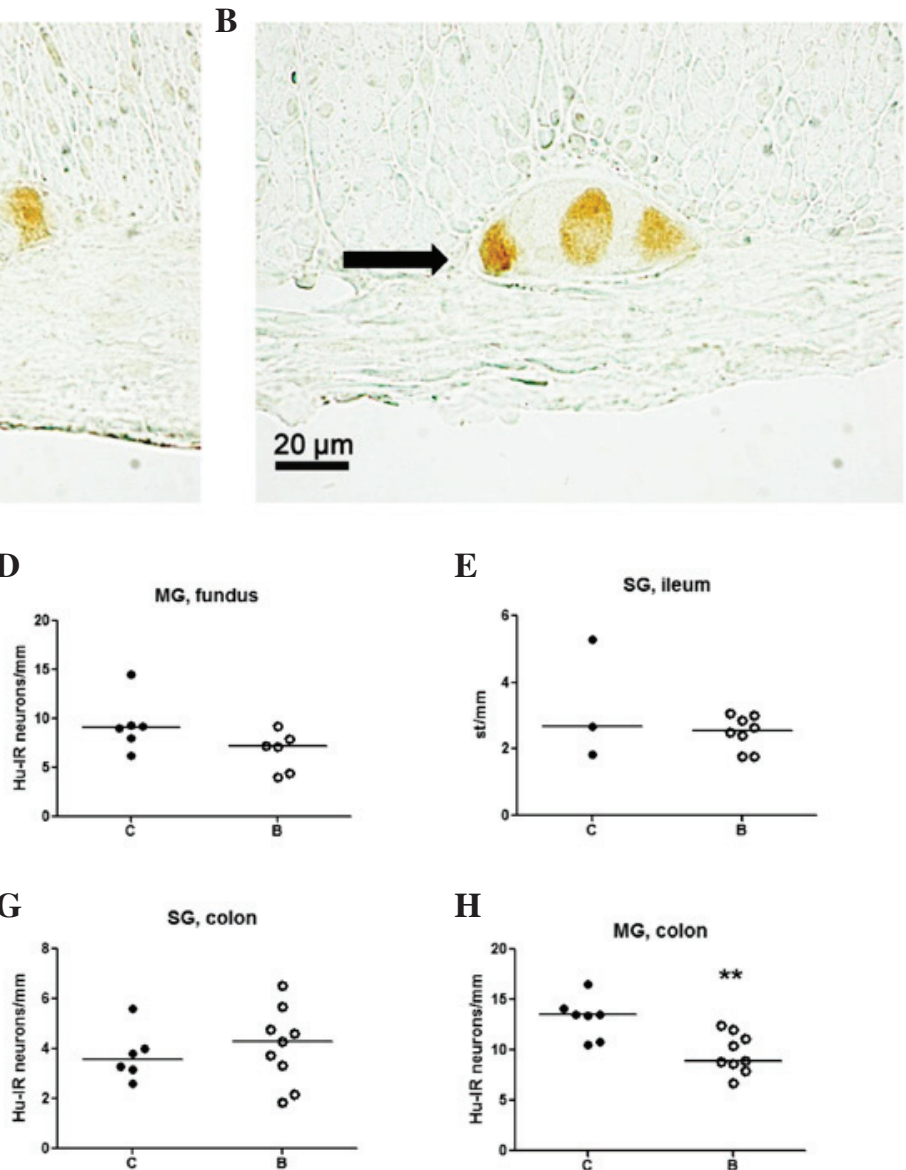

E

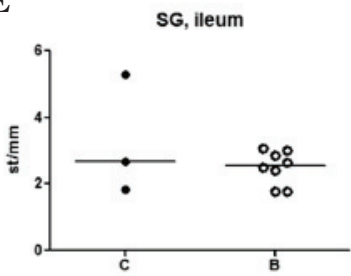

H

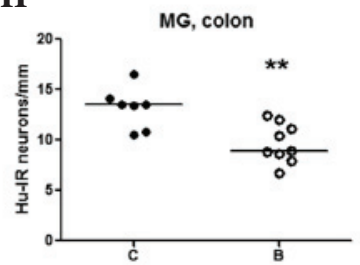

Figure 3. Paraffin sections of MG in the colon from rats treated with (A) saline or (B) buserelin, stained with primary antibodies raised against the human neuronal protein (HuC/D). Arrows indicate myenteric ganglia. Scale bar, $20 \mu \mathrm{m}$. Numbers of neurons in the (C) SG and (D) MG in the fundus, (E) SG and (F) MG in the ileum, and (G) SG and (H) MG in the colon of rats treated with saline or buserelin. Cells were counted in longitudinally cut whole-wall sections. Rats treated with buserelin showed decreased numbers of myenteric neurons in the ileum and colon compared with the controls. $\mathrm{C}=3-7$ rats and $\mathrm{B}=6-9$ rats. Results are presented as individual data and medians and analyzed using Mann-Whitney U-test; ${ }^{* *} \mathrm{P}<0.01$, compared with control. SG, submucosal ganglia; MG, myenteric ganglia; C, control; B buserelin-treated.

The number of T-lymphocytes was significantly increased in the submucosa $(\mathrm{P}<0.01)$ (Fig. 6A) and circular muscle layer of fundus $(\mathrm{P}<0.05)$ (Fig. 6B) in the buserelin-treated group, as compared with the controls.

In the ileum, no significant changes in the numbers of T-lymphocytes in the submucosa or circular muscle layer were detected between the two groups (Fig. 6C and D). In the colon, the number of T-lymphocytes in the submucosa did not differ between the two groups, whereas in the circular muscle layers, the numbers of T-lymphocytes were increased in the buserelin-treated group, as compared with the controls $(\mathrm{P}<0.05)$ (Fig. 6E and F). 
A

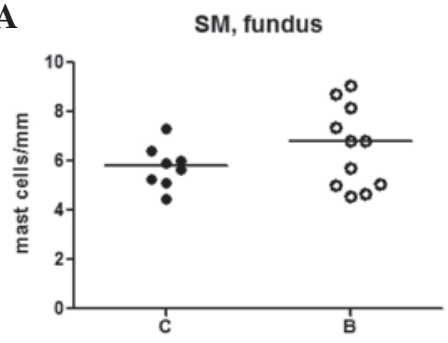

SM, ileum

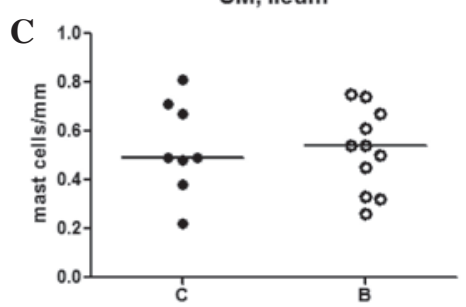

B

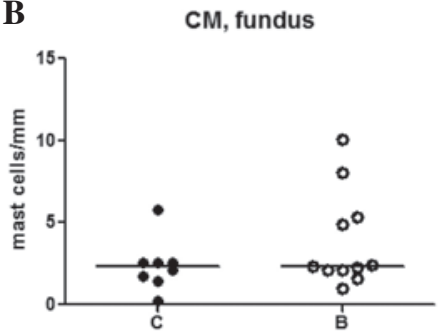

SM, colon

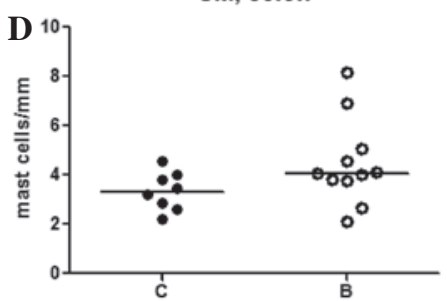

Figure 4. Number of mast cells analyzed in the (A) SM and (B) CM in the fundus, and in the SM of the (C) ileum and (D) colon, between rats treated with saline or buserelin. Cell counting was performed on cross- and longitudinally cut, whole-wall sections. $\mathrm{C}=8$ rats and $\mathrm{B}=11$ rats. Results are presented as individual data and medians. Mann-Whitney U-test was used for analysis. SM, submucosa; CM, circular muscle layer; C, control treated with saline; B, buserelin.

A

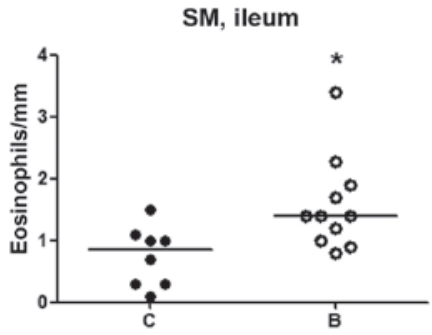

B

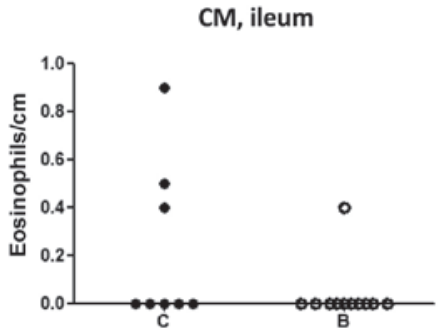

C

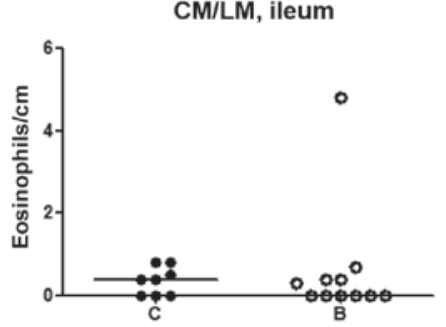

Figure 5. Numbers of eosinophils in the (A) SM, (B) CM and (C) between the CM and LM of the ileum in rats treated with saline or buserelin. Cell counting was performed on cross- and longitudinally cut whole-wall sections. Buserelin-treated rats had increased presence of eosinophils in the submucosa of ileum. $\mathrm{C}=8$ rats and $\mathrm{B}=11$ rats. Results are presented as individual data and medians and analyzed by Mann-Whitney $\mathrm{U}$-test; ${ }^{*} \mathrm{P}<0.05$, compared with control. SM, submucosa; CM, circular muscle layer; LM, longitudinal muscle layer; C, control treated with saline; B, buserelin.

A

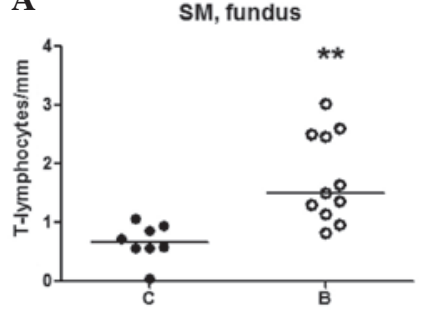

D

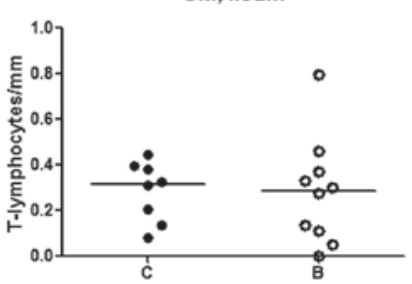

B
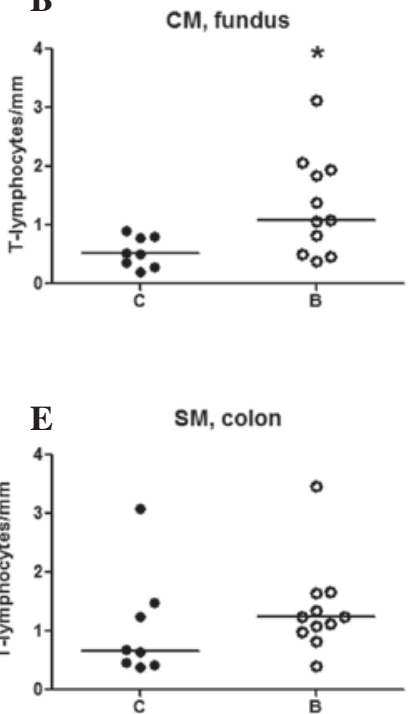

C
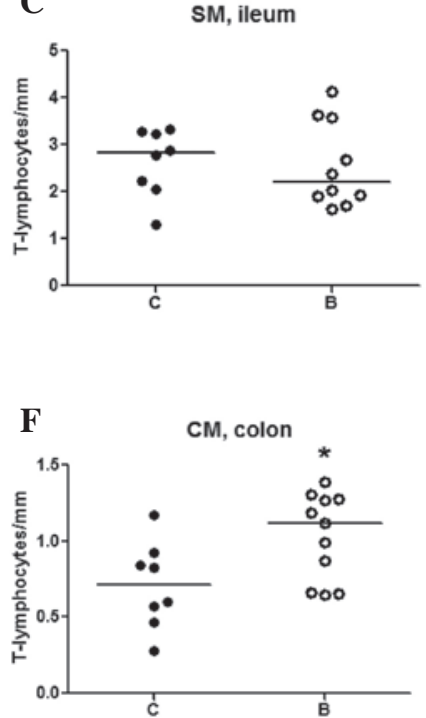

Figure 6. Numbers of T-lymphocytes in the (A) SM and (B) CM in the fundus, in the (C) SM and (D) CM in the ileum and in the (E) SM and (F) CM in the colon in rats treated with saline or buserelin. Cell counting was performed on cross- and longitudinally cut whole-wall sections. Rats treated with buserelin showed an increased number of T-lymphocytes in the $\mathrm{SM}$ and $\mathrm{CM}$ of the fundus, and the $\mathrm{CM}$ of thecolon, compared with controls. $\mathrm{C}=8$ rats and $\mathrm{B}=11$ rats. Results are presented as individual data and medians and analyzed by Mann-Whitney U-test; ${ }^{*} \mathrm{P}<0.05$ and ${ }^{* *} \mathrm{P}<0.01$ compared with control. SM, submucosa; CM, circular muscle layer; C, control treated with saline; B, buserelin. 


\section{Discussion}

Reduced numbers of myenteric neurons in the ileum and colon were detected in buserelin-treated rats compared with controls in the present long-term study. Buserelin treatment resulted in a lower body weight at the end of the study, a thinner circular muscle layer in the ileum, a thinner longitudinal muscle layer in the colon, an increased number of eosinophils in the submucosa of the ileum, and a higher number of T-lymphocytes in the submucosa of the fundus, and circular muscle layers of the fundus and colon, compared with controls.

Sixteen weeks after the last treatment session, at study week 32 , the rats treated with buserelin showed a loss of myenteric neurons in the ileum and colon, as described in previous studies where the rats were sacrificed shortly after the end of the treatment $(4,10)$. In accordance with former studies, the neuronal loss was more pronounced in the ileum and colon than in the fundus, and in myenteric neurons than in the submucosal neurons $(4,10)$. Regeneration of enteric neurons and glial cells is possible, since tissue-specific progenitor cells have been identified $(16,17)$, and mice studies have shown regeneration of neurons following intestinal damage (18). However, the magnitude of myenteric neuronal loss was the same as that determined in previous studies $(4,10)$, even though the present rats were kept alive for 16 weeks longer.

The difference in body weight between groups started at week 23, and was not observed in the short-term studies that ended at week 16 and $18(4,10)$, which suggests that the damage that occurs during the treatment eventually leads to malnutrition. The enteric nervous system (ENS) has an impact on digestion and absorption of nutrients, particularly carbohydrates and fat $(19,20)$, and an increased fat content in the feces following buserelin treatment has been observed (10). Enteric neuropathy may thus lead to malnutrition in rats as well as in patients $(3,9)$. Sex hormones stimulate increased food intake, and low plasma estradiol levels have been shown to result in reduced food intake (21). In a previous study, no difference in body weight was identified; however, plasma levels of estradiol were elevated and the uterine muscle layer was thickened following buserelin treatment (10). The unaffected myometrium thickness in the present study suggests normalized sex hormone levels following treatment cessation, and the negative effects of neuropathy on intestinal nutrient uptake cannot be counteracted by increased appetite and food intake secondary to elevated sex hormone levels, as an assumed explanation to the reduced body weight in the present long-term follow-up.

In previous studies, buserelin caused a loss of myenteric neurons with signs of apoptosis, but without signs of muscle degeneration and increased eosinophils $(4,10)$. In previous studies, the number of T-lymphocytes was only increased in the ganglia and around nerve fibers $(4,11)$. This indicates that neuronal loss is the initial damage, and the other findings are secondary. Degenerative neuropathy may result in secondary, and not causal, tissue inflammation. A variety of diseases in the human GI tract are accompanied by eosinophilia, which is an un-specific condition, without obvious consequences (22). Mucosal eosinophilia is associated with hypersensitivity (23), but this has not been shown for mural forms (24).
T-lymphocytes are involved in the development and activity of inflammatory bowel disease (25), and antibodies against CD3 have been used to prevent immunological diseases (26). Although a number of patients with ENS diseases express an increased number of T-lymphocytes in the bowel wall, and in these patients T-lymphocytes have been shown in close relation to ganglia, their role in enteric neuropathy is not defined $(10,27)$. The increased number of T-lymphocytes in the fundus, albeit normal numbers of neurons in the present study, may be explained by a dynamic process over time, and when examining the rats at a specific time point, the process may be at different stages in different bowel segments. The increased number of T-lymphocytes may have been proceeded by reversible neuronal damage. The thinner muscle layer in the intestines could be explained by less neuronal stimulation and less peristalsis, resulting in atrophy of the muscle.

The clinical course of CIPO and ED is a slow propagation of the disease over time with worsened GI functions $(3,9,27)$. These conditions show varying histopathological changes with preserved submucosal neurons and affected myenteric ganglia $(3,9,27)$, similar to the rat model. Enteric neuropathies may be accompanied with muscle hypertrophy or muscle atrophy (27). It is of great interest to examine the long-term effects of enteric neuropathy in a well-characterized and standardized rat model, as it is difficult to study neuropathies in humans due to the rarity of the diseases and poor sensitivity of GI function tests. In addition, examination of ENS demands a full-thickness biopsy obtained through surgery. Furthermore, there are different etiologies to the ENS damage, and different co-morbidities and life style habits that also have an effect on these patients. Several confounding factors are therefore at hand in clinical studies, which obscure the results rendering it difficult to determine which are primary etiological effects, and which are secondary to the evoked disturbances. Taken together, all clinical studies performed on buserelin-induced intestinal damage in humans, and experimental studies in rats, suggest that this neuropathy may be classified as a primary degenerative or inflammatory neuropathy $(3,4,9-11)$.

In conclusion, long-term follow-up of buserelin-induced enteric neuropathy in a rat model shows irreversible loss of myenteric neurons. Secondary to this, the histopathological GI changes were aggravated over time and the rats presented with thinner muscle layers, and increased numbers of eosinophils and T-lymphocytes. In addition, the body weight gain is reduced over time. Future mechanistic studies are necessary in order to explain the pathophysiology underlying enteric neuropathy and the associated changes.

\section{Acknowledgements}

The King Gustaf V and Queen Victoria Free Mason's Foundation, Development of Region Skåne, the Bengt Ihre Foundation, the Lundström Foundation, and the Royal Physiographic Society in Lund. The authors would like to thank Anna Themner Persson for her technical assistance.

\section{References}

1. Naor Z: Signalling by G-protein-coupled receptor (GPCR): Studies on the GnRH receptor. Front Neuroendocrinol 30: 10-29, 2009. 
2. Huang W, Yao B, Sun L, Pu R, Wang L and Zhang R: Immunohistochemical and in situ hybridization studies of gonadotropin releasing hormone $(\mathrm{GnRH})$ and its receptor in rat digestive tract. Life Sci 68: 1727-1734, 2001.

3. Ohlsson B, Veress B, Janciauskiene S, Montgomery A, Haglund $M$ and Wallmark A: Chronic intestinal pseudo-obstruction due to buserelin-induced formation of anti-GnRH antibodies. Gastroenterology 132: 45-51, 2007.

4. Sand E, Voss U, Hammar O, Alm R, Fredrikson GN, Ohlsson B and Ekblad E: Gonadotropin-releasing hormone analog buserelin causes neuronal loss in rat gastrointestinal tract. Cell Tissue Res 351: 521-534, 2013.

5. Chen L, He HX, Sun XD, Zhao J, Liu LH, Huang WQ and Zhang RQ: Expression of gonadotropin-releasing hormone receptor and effect of gonadotropin-releasing hormone analogue on proliferation of cultured gastric smooth muscle cells of rats. World J Gastroenterol 10: 1780-1784, 2004.

6. Aguilar-Rojas A and Huerta-Reyes M: Human gonadotropin-releasing hormone receptor-activated cellular functions and signaling pathways in extra-pituitary tissues and cancer cells (Review). Oncol Rep 22: 981-990, 2009.

7. Ducker TE, Boss JW, Altug SA, Mehrabian H, Dekeratry DR, Clench MH and Mathias JR: Luteinizing hormone and human chorionic gonadotropin fragment the migrating myoelectric complex in rat small intestine. Neurogastroenterol Motil 8: 95-100, 1996.

8. Sand E, Voss U, Ohlsson B and Ekblad E: Luteinizing hormone receptors are expressed in rat myenteric neurons and mediate neuronal loss. Auton Neurosci 193: 104-107, 2015.

9. Cordeddu L, Bergvall M, Sand E, Roth B, Papadaki E, Li L, Damato $\mathrm{M}$ and Ohlsson B: A description of cases with severe abdominal complaints after treatment with gonadotropin-releasing hormone analogs. Scand J Gastroenterol 16: 1-9, 2015.

10. Sand E, Roth B, Weström B, Bonn P, Ekblad E and Ohlsson B: Structural and functional consequences of buserelin-induced enteric neuropathy in rat. BMC Gastroenterology 14: 209, 2014.

11. Ohlsson B, Sand E and Veress B: Ganglioneuritis is common in rats with enteric neuropathy due to buserelin treatment. Regul Pept 190-191: 43-45, 2014.

12. Sasson R, Rimon E, Dantes A, Cohen T, Shinder V, Land-Bracha A and Amsterdam A: Gonadotrophin-induced gene regulation in human granulosa cells obtained from IVF patients. Modulation of steroidogenic genes, cytoskeletal genes and genes coding for apoptotic signalling and protein kinases. Mol Hum Reprod 10: 299-311, 2014.

13. Trindade CR, Camargos AF and Pereira FE: The effect of buserelin acetate on the uterus of adult rats: Morphological aspects. Clin Exp Obstet Gynecol 35: 198-201, 2008.
14. Sand E, Themner-Persson A and Ekblad E: Mast cells reduce survival of myenteric neurons in culture. Neuropharmacology 56 : 522-530, 2009.

15. Sand E, Themner-Persson A and Ekblad E: Infiltration of mast cells in rat colon is a consequence of ischemia/reperfusion. Dig Dis Sci 53: 3158-3169, 2008

16. Kawaguchi J, Nichols J, Gierl MS, Faial T and Smith A: Isolation and propagation of enteric neural crest progenitor cells from mouse embryonic stem cells and embryos. Development 137: 693-704, 2010.

17. Heanue TA and Pachnis V: Prospective identification and isolation of enteric nervous system progenitors using Sox2. Stem Cells 29: 128-140, 2011.

18. Laranjeira C, Sandgren K, Kessaris N, Richardson W, Potocnik A, Vanden Berghe P and Pachnis V: Glial cells in the mouse enteric nervous system can undergo neurogenesis in response to injury. J Clin Invest 121: 3412-3424, 2011.

19. Ratnayake WM and Galli C: Fat and fatty acid terminology, methods of analysis and fat digestion and metabolism: A background review paper. Ann Nutr Metab 55: 8-43, 2009.

20. Mourad FH and Saadé NE: Neural regulation of intestinal nutrient absorption. Prog Neurobiol 95: 149-162, 2011.

21. Hirschberg AL: Sex hormones, appetite and eating behaviour in women. Maturitas 71: 248-256, 2012.

22. Yantiss RK: Eosinophils in the GI tract: How many is too many and what do they mean? Mod Pathol (28 Suppl 1): S7-S21, 2015.

23. Rothenberg ME: Eosinophilic gastrointestinal disorders (EGID). J Allergy Clin Immunol 113: 11-28, 2004.

24. Yun MY, Cho YU, Park IS, Choi SK, Kim SJ, Shin SH and Kim KR: Eosinophilic gastroenteritis presenting as small bowel obstrction: A case report and review of the literature. World J Gastroenterol 13: 1758-1760, 2007.

25. Forster K, Goethel A, Chan CW, Zanello G, Streutker C and Croitoru K: An oral CD3-specific antibody suppresses T-cell-induced colitis and alters cytokine responses to T-cell activation in mice. Gastroenterology 143: 1298-1307, 2012.

26. Ochi H, Abraham M, Ishikawa H, Frenkel D, Yang K, Basso A, Wu H, Chen ML, Gandhi R, Miller A, et al: New immunosuppressive approaches: Oral administration of CD3-specific antibody to treat autoimmunity. J Neurol Sci 274: 9-12, 2008.

27. Knowles CH, De Giorgio R, Kapur RP, Bruder E, Farrugia G, Geboes K, Gershon MD, Hutson J, Lindberg G, Martin JE, et al: Gastrointestinal neuromuscular pathology: Guidelines for histological techniques and reporting on behalf of the gastro 2009 International working group. Acta Neuropathol 118: 271-301, 2009. 